\title{
Meta-analysis on maternal deaths and assessing significant factors for heterogeneity between states of India during 2016
}

\author{
Vanamail Perumal* \\ Department of Obstetrics and Gynaecology, All India Institute of Medical Sciences, Ansari Nagar, New Delhi, India
}

Received: 03 June 2021

Accepted: 06 July 2021

\section{*Correspondence:}

Dr. Vanamail Perumal,

E-mail: pvanamail@gmail.com

Copyright: $\odot$ the author(s), publisher and licensee Medip Academy. This is an open-access article distributed under the terms of the Creative Commons Attribution Non-Commercial License, which permits unrestricted non-commercial use, distribution, and reproduction in any medium, provided the original work is properly cited.

\begin{abstract}
Objective of the study was to provide a more precise estimate of maternal mortality (MM), maternal mortality ratio (MMR) and to identify significant factors contributing for heterogeneity between the states in India. "Metaprop" procedure in STATA software, which are specific to binomial data was applied on state wise MM data published by sample registration system (SRS) during 2014-16. An overall MM estimate and potential sources of heterogeneity could be identified using meta-regression. Corrected estimates of MMR by states were compared. SRS published the MM data by 17 Major states. Overall reported MM was 8.8 per 100, 000 women. Estimate obtained by random effect model was 8.3 (95\% CI: 5.9-11.1) per 100,000 women. Heterogeneity between states was very high $\left(\mathrm{I}^{2}\right.$-statistics $\left.=91.9 \%\right)$, and egger regression revealed no reporting bias $(\mathrm{p}=0.672)$. Meta-regression analysis indicated that the percent women attending full antenatal care (ANC) visits found to be highly significant $(\mathrm{p}<0.001)$ for MM with inverse relationship implying that the states with a higher percentage of women with full ANC visits are likely to have lesser MM. While the estimate of MMR by SRS was 130 per 100,000 live births, corrected MMR was 123 (95\% CI: 87-164) accounting for $26 \%$ reduction from previous estimate 167 obtained in 2013. This paper provided a precious estimate of both MM and MMR adjusted for sampling weight. Further, the importance of either full ANC visits or four ANC visits could be demonstrated for reduction in MMR on achieving the Millennium development goal (MDG) in the country.
\end{abstract}

Keywords: MM, MMR, Meta-analysis, ANC visits, India

\section{INTRODUCTION}

Maternal health is one of the health indicators to assess the quality of well-being in any country. Post-partum survival status of both mother and child is playing a major role in the quality of health services in the country. During the period 1990-2015, MM worldwide dropped by about $44 \%$. Between 2016 and 2030, as part of the sustainable development goals, the target is to reduce the worldwide MMR to less than 70 per 100,000 live births. ${ }^{1}$ According to worldwide estimate of MM during 2015 India contributed about $19 \%$ of MM annually to the global annual estimate $(358,000)$. Considering this alarming situation, millennium development goals (MDG) 4 (reduction in child mortality between 1990 and 2015) and 5 (reduction in MM) were set as the target in India. ${ }^{2}$ To achieve MDG-5 a target for MMR was fixed at 109 per 100,000 live births. ${ }^{3}$ An earlier study highlighted that though some progress was made in achieving the MDG-5, due to limited resources allotted to adolescents, the poor, and women living in rural areas the target was seemed to be unattainable. ${ }^{4}$

Due to implementation of various maternal health programmes, India has made remarkable progress in reducing $\mathrm{MM}$ in the last two decades. Parallel implementation of improving child health program significantly increased the live birth rate, and both these two indicators reduced the MMR, while global MMR was 400 during the year 1990, India recorded 600 in the same period. In the next two decades period, the corresponding figures were 210 and 178 respectively and accounting for 
$70 \%$ reduction in India over a period of 20 years. After implementation of various activities under national rural health mission (NRHM) program, annual reduction rate in MMR was shown to be an increasing trend from $4.1 \%$ during $2001-2003$ to $5.7 \%$ in $2010-12$. However, the corresponding reduction rate in the same period at global level was $2.4 \%$. All the estimates in India were made using data of registrar general and census commissioner, India, sample registration system (SRS). ${ }^{5}$ Though these estimates were made using uniform sampling technique. Model based significant factors that are likely to contribute for MMR were not studied in India till the date. Therefore, present study was carried out with the following objectives-a) To provide a more precise estimate of MM rate in India and b) To assess significant variables that were likely to contribute for heterogeneity in MMR between various states.

\section{METHODS}

\section{Source}

SRS is the responsible organization to provide direct estimates of MM through a nationally representative sample on a periodic basis. The latest bulletin provided the levels of MM and MMR for the period 2014-16. ${ }^{6}$ For better understanding of the MM and MMR situation in the country, the SRS presented the data by major states in the country, and these indicators were considered as outcome variables. To find out possible significant variables that may be associated with MM, following variables in the reproductive age group (15-19 years) formed covariate-a) Percentage of women with $\geq 10$ years of schooling, b) Percentage of married women before 18 years of age. b) Percentage of women married or pregnant in 15-19 years, c) Percentage of women using pills, d) Percentage of women with total unmet need, e) Percentage of women with unmet need for spacing, f) Percentage of women undergone first ANC visit, g) Percentage of women undergone four ANC visits, h) Percentage of women received mother and child protection card and i) Percentage of women gave birth in public health facility

National family health survey (NFHS-4) reports available for 2015-16 period provided all these covariates. ${ }^{7}$

\section{Statistical analysis}

Meta-analysis for proportion of MM were carried out by considering state wise data as study identification. All statistical analyses were performed using statistical software STATA version 14.0 (Stata Corp, College Station, TX, USA). A statistical program employed to carry out meta-analyses of proportions in STATA $^{8}$ is "Metaprop" and it is an extension of "metan" procedure, which is used to get pool estimate of risk ratios, odds ratios, differences of risks or means. "Metapro" procedures are specific to binomial data and allow computation of exact binomial confidence intervals. It provides appropriate methods for dealing with proportions close to or at the margins where the normal approximation procedures deviates, by use of the binomial distribution to model the within-study variability or by allowing Freeman-Tukey double arcsine transformation to stabilize the variances. Since proportion of MM is close to zero in the present study, "Metaprop" procedure is appropriate. To decide adequate model of fixed effect/random effect, heterogeneity measure formed the criteria. Value of $\mathrm{I}^{2}$ index derived from Q-Cochrane was set to be $<25 \%$ for homogeneity. Based on appropriate model forest plot was implemented for showing the results of the individual and pooled effects with $95 \%$ confidence interval (CI) of all the states. To evaluate publication bias, both graphical presentation (Funnel plot) and statistical test (Egger's test) were carried out. In addition, to identify variables contributing for heterogeneity between states univariate meta-regression analyses were carried out. Variables found to be significant with $\mathrm{p}<0.2$ formed covariates for multivariable meta-regression analysis. Two-sided $\mathrm{p}<0.05$ for all statistical tests considered to the significant.

\section{RESULTS}

SRS organization carried out the survey on MM during 2014-16 through the nationally representative sample. All the reported deaths during the survey were confirmed by autopsy instruments. Along with maternal deaths details of live births were also recorded. The survey data were presented by 17 major states and classified into three categories (South, empowered action group-Assam and other states). Total 556 maternal deaths were recorded (EAG) out of 62, 96,101 women of reproductive age group (15-49 years) surveyed, accounting for 8.8 (95\% CI: 8.19.5) MM per 100,000 women. MMR estimated from these 556 maternal deaths and 4,26,861 live births was 130 per 100,000 live births.

Meta-analysis results showed heterogeneity measure $\left(\mathrm{I}^{2}-\right.$ index $=91.9 \%$ ) between the states was more than cut-off value $(<25 \%)$. Therefore, fitting of a random effect model resulted in an overall estimate of MM 8.3 (95\% CI: 5.911.1) per 100,000 women. State wise estimate of MM with 95\% CI is depicted as forest plot in Figure 1. According to classification of states by categories, the estimate for south states is shown to be with the least MM (4.5; 95\% CI: 2.76.8) and in EAG-Assam states revealed maximum MM (15.9; 95\% CI: 14.3-17.7). In the other states, category MM is shown to be 5.4 (95\% CI: 4.4-6.6). Since maternal death proportion was in the extreme level $(\approx 0)$, as suggested in an earlier study funnel plot was obtained for logit value of effect size (MM) and sample size. ${ }^{9}$ Funnel plot showed to be symmetric and implying that due to implementation of uniform sampling protocol no reporting bias revealed. In addition, Egger regression analysis also revealed that there was no reporting bias $(\mathrm{p}=0.672)$. Univariate meta-regression analysis was carried out to identify significant variables that contributed for heterogeneity and presented in Table 1 . It is observed that the percent women attending full ANC visits (full ANC is, at least four ANC visits, at least one tetanus toxoid (TT) 
injection and iron folic acid tablets or syrup taken for 100 or more days) during pregnancy was found to be highly significant variable $(\mathrm{p}<0.001)$, which explained about $87 \%$ variation in $\mathrm{MM}$ followed by four ANC visit $(83 \%$ variation) and one ANC visit (56\% variation). Percent of women with more than 10 years of schooling also emerged as significant variable (41\% variation) for MMR. Variables such as percent of women with more than 10 years of schooling, percent of women with total unmet, percent of women with full ANC visit and percent of women gave birth in public health facilities were significant at $p<0.2$ and eligible for inclusion in the multivariable meta-regression analysis. Of these variables, percent of women with full ANC visit emerged as significant variable $(\mathrm{p}<0.01)$ with $85 \%$ variation. Predicted MM against full ANC visit is shown in Figure 2. It can be implied that while achieving $60 \%$ full ANC visit the expected MM is likely to be around two per 100,000 population.

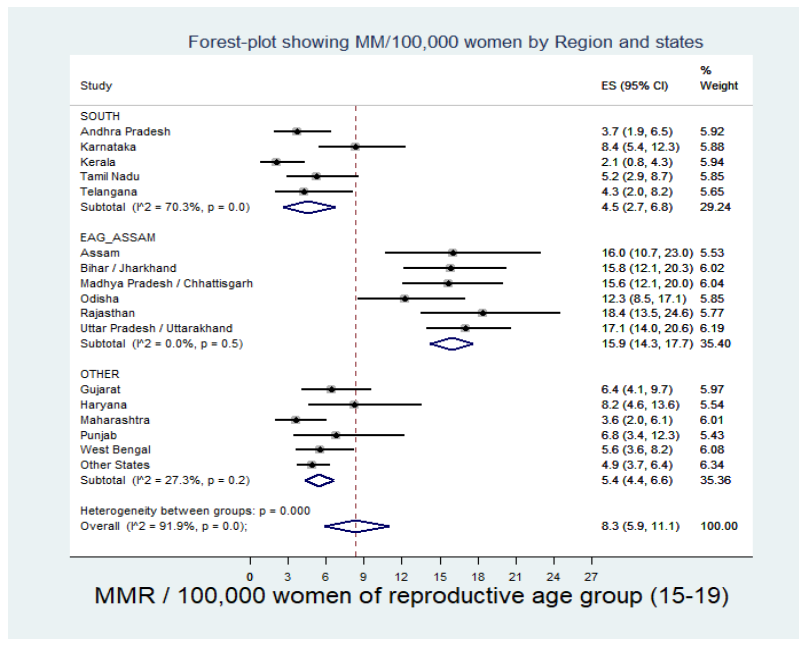

Figure 1: MMR/1000,000 women of reproductive age group (15-19).

Table 1: Univariate analysis of meta-regression to determine significant variables for heterogeneity between states.

\begin{tabular}{|llllll|}
\hline Variables & $\begin{array}{l}\text { Regression } \\
\text { coefficient }\end{array}$ & $\mathbf{S E}$ & $\begin{array}{l}\mathbf{P} \\
\text { value }\end{array}$ & $\begin{array}{l}\text { Heterogeneity } \\
\text { measure }\left(\mathbf{I}^{2}\right)\end{array}$ & $\begin{array}{l}\text { Explained } \\
\text { variation }\left(\mathbf{R}^{2}\right)\end{array}$ \\
\hline Schooling $>\mathbf{1 0}$ years & -0.034 & 0.010 & 0.006 & 87.8 & 41.3 \\
\hline Married before age 18 years & 0.021 & 0.017 & 0.219 & 91.1 & 3.8 \\
\hline Mothers at age 15-19 years & -0.001 & 0.041 & 0.985 & 91.46 & 0.0 \\
\hline Women use pills & 0.021 & 0.023 & 0.392 & 91.6 & 0.0 \\
\hline Women with total unmet & 0.061 & 0.035 & 0.100 & 90.59 & 13.9 \\
\hline Women with unmet spacing & 0.047 & 0.081 & 0.569 & 91.62 & 0.0 \\
\hline Women with first ANC visit & -0.042 & 0.010 & 0.001 & 83.21 & 56.5 \\
\hline Women with four ANC visit & -0.029 & 0.004 & 0.001 & 65.35 & 82.7 \\
\hline Women with full ANC visit & -0.044 & 0.006 & 0.001 & 56.45 & 87.2 \\
\hline Women with mother child protection & 0.022 & 0.040 & 0.588 & 91.6 & 0.0 \\
\hline Women gave birth in public health facilities & 0.021 & 0.013 & 0.080 & 91.6 & 12.6 \\
\hline
\end{tabular}

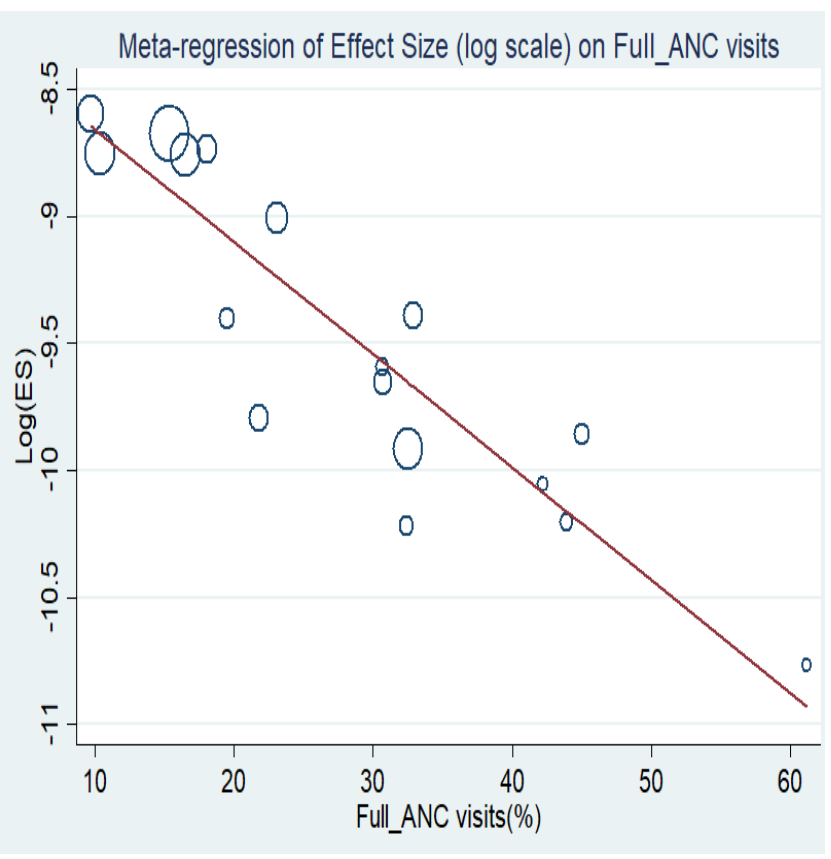

Figure 2: Meta-regression of effect size (10\% scale) on full ANC visits.

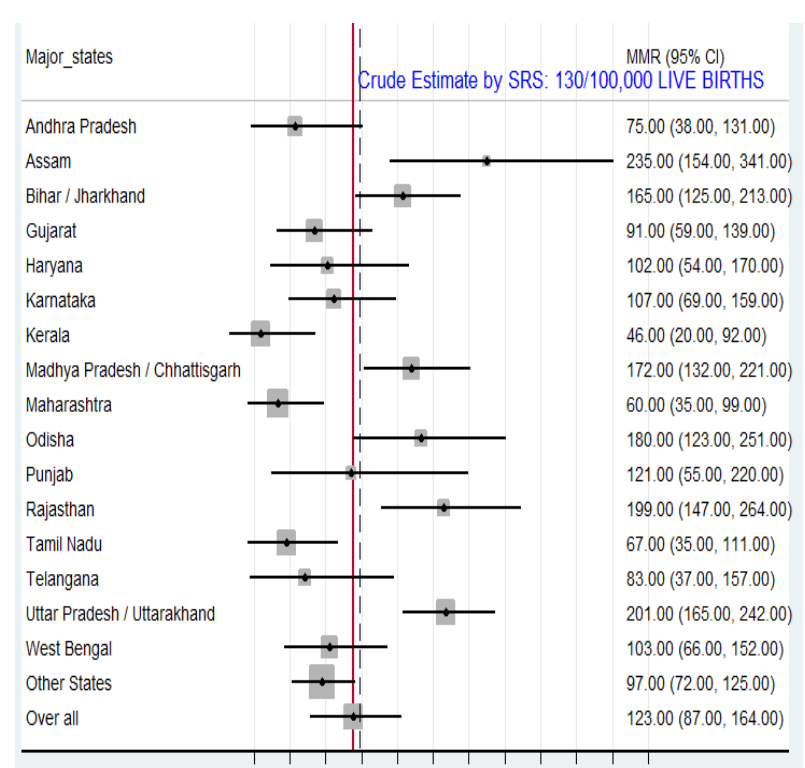

4070100130160190220250280310340370 MATERNAL MORTALITY RATIO/100,000 LIVE BIRTHS

Figure 3: MMR/100,000 live births. 
Using state wise estimate of MM corrected MMR is shown in forest plot (Figure 3). It is observed that the overall estimate of MMR is found to be 123 (95\% CI: 87-164) per 100, 000 live births. Among 17 major states estimates of 11 states showed less than the National estimate. Interestingly of these 11 states, estimates of five states showed less than the lower limit of national estimate. All the six states in which, estimates were more than the national estimate are from North India. On examining the percent of women with full ANC visit in these states, the average was abysmally low (19\%) as compared to $34 \%$ in the 11 states in which MMR was less than the national estimate. Similarly, in those six hotspot states percent of women who had home delivery and anemia during pregnancy was $5.1 \%$ and $48.9 \%$ respectively. The corresponding figures for 11 states were $3.1 \%$ and $46 \%$.

\section{DISCUSSION}

Performing meta-analysis to get precious estimate of MM may be the first in India, which contributes about $18 \%$ of the total world population. After adjusting for sampling weight, the overall estimate of MM was 8.3 per 100,000 women of reproductive age groups. Though there was no marked difference between the meta-analysis estimate and the estimate made by SRS (8.8), the decline rate 5.7\% (8.8 to 8.3) made significant contribution in the MMR estimate. On account of this decline rate, the corrected MMR was found to be 123 per 100, 000 live births as compared to 130 by SRS. The corrected MMR contributed a significant reduction (26.3\%) from the earlier estimate of MMR (167) obtained during 2011-2013. ${ }^{6}$ This phenomenal reduction over a period of 2 years certainly might be due to regular monitoring of maternal health indicators such as antenatal check-up, institutional delivery and delivery performed by trained and skilled personnel, and post-natal care through health management information system (HMIS) as highlighted in the SRS bulletin. ${ }^{10}$ Simultaneous implementation of free service guarantee at public health facilities might also be another factor for recording higher institutional deliveries to reduce MMR. However, an earlier study pointed out that evaluating the extent of progress towards MDG-5 is an uphill task due to the lack of reliable and accurate $\mathrm{MM}$ data in developing-country like India, where MM is high. ${ }^{11}$ Therefore, as a part of monitoring the progress towards MDG-5 UN MM estimation inter-agency group (UN MMEIG) estimated MMR during the year 2015, and it was 174 per 100,000 livebirths against 130 made by SRS in the year 2016. The difference might be due to the time gap between the two estimates and however, the relative reduction from the previous estimates reflects the active monitoring of women and child health indicators by national rural health mission (NRHM). Achieving the target, MDG-5 was proved in one of the states (Tamil Nadu) by intensive implementation and monitoring of NRHM activities. ${ }^{12}$

Present analysis could identify hotspot states in which MMR was more than the National estimate. Factors such as very low compliance rate of full ANC visit, high percentage of women with home delivery and higher anemia level during pregnancy in these states might have contributed for higher MMR. Of these factors, the importance of full ANC visit was established in reducing MMR. If all the states in India ensured for at least $60 \%$ full ANC visit, achieving MDG-5 will be possible at least in the next five years. Further, importance of institutional delivery in reducing MMR was demonstrated in an earlier study in which community-based intervention support for promoting institutional deliveries resulted in an annual reduction of $17 \%$ in MM. ${ }^{13}$ Cash incentive scheme-Janani Suraksha Yojana (JSY) initiated by government of India to promote institutional deliveries also proved to be very effective. ${ }^{14-17}$ Similarly, community-based behaviour change management intervention program also demonstrated the improvement of maternal health quality. ${ }^{18}$

Since India is a country of multi-culture with different ecological zones and diversified socio-economic conditions, there will a considerable variation in accessing safe mother-hood. In the present analysis percentage of women with at least 10 years of education was found to be a significant factor in reducing the MM implying that the educated mothers are likely to have safe mother-hood by availing at least four ANC visits if not Full ANC. The importance of education was also highlighted in an earlier study, stating that the odds for a safe pregnancy were three times higher for educated women compared with a base case of no education. ${ }^{19}$

Though percentage of institutional deliveries is significantly improved to more than $95 \%$ over all in the country, current sluggish rate of decline in MMR indicates that MMR reduction is not only a function of hospitalbased approaches and cash incentive programme. Apart from these programmes, a novel approach should be initiated to enhance ANC visits and to reduce MM. India is progressing well in the field of communication network due to the active role of the private and public network system. According to telecom authority of India teledensity was $93 \%$ as on March $2017 .{ }^{20}$ Therefore, using the revolution of the communication system, creating awareness about preventable causes of maternal death through mobile-based social media such as WhatsApp, Facebook and Twitter should be undertaken by government and civil society. Further, "apps" may be developed, and all pregnant mothers may be informed of their ANC visit schedule, TT-injection schedule and also consequences of non-compliance may be revealed.

\section{CONCLUSION}

Through meta-analysis a precious estimate of MMR could be demonstrated in India. Further, the importance of ANC visits for significant reduction in MMR was established. If these observations are taken care at programme level through social media in an intensive mode, achieving the target of MDGs will be possible at least in the next 5 years. 


\section{ACKNOWLEDGMENTS}

The author would like to thanks registrar general, census of India, NFHS-4 nodal agencies (International institute of population sciences (IIPS), Mumbai, India and ministry of health and family Welfare, India) for accessing data from their public domain.

\section{Funding: No funding sources \\ Conflict of interest: None declared \\ Ethical approval: Not required}

\section{REFERENCES}

1. Hogan MC, Foreman KI, Naghavi M, Ahn SY, Wang M, Makela SM et al. Maternal mortality for 181 countries, 1980-08: a systematic analysis of progress towards Millennium Development Goal 5. Lancet. 2010;375:1609-23.

2. Chatterjee A, Paily VP. Achieving Millennium Development Goals 4 and 5 in India. BJOG. 2011;118(2):47-59.

3. Trends in Maternal Mortality: 1990 to 2008. Estimates developed by WHO, UNICEF, UNFPA and The World Bank. World Health Organisation, 2010. Available

at: http://www.whqlibdoc.who.int/publications/2010/978 9241500265_eng.pdf. Accessed on 10 Jan 2020.

4. Rai RK, Tulchinsky TH. Addressing the sluggish progress in reducing maternal mortality in India. Asia Pac J Public Health. 2015;27(2):NP1161-9.

5. Census of India-Vital Statistics-Sample Registration System. Available at: http://censusindia.gov.in/vital_statistics/SRS/Sample_ Registration_System.aspx. Accessed on 10 Jan 2020.

6. Special bulletin on maternal mortality in India 201416. Available at: http://censusindia.gov.in/vital_statistics/SRS/Sample_ Registration_System.aspx. Accessed on 2018 Feb 26

7. National Family Health Survey 2015-16 (NFHS-4)state fact sheet.pdf. Available at: http://rchiips.org/NFHS/factsheet_NFHS-4.shtml. Accessed on 2018 Feb 26.

8. Nyaga VN, Arbyn M, Aerts M. Metaprop: a Stata command to perform meta-analysis of binomial data. Arch Public Health. 2014;72:39.

9. Hunter JP, Saratzis A, Sutton AJ, Boucher RH, Sayers $\mathrm{RD}$, Bown MJ. In meta-analyses of proportion studies, funnel plots were found to be an inaccurate method of assessing publication bias. $\mathrm{J}$ Clin Epidemiol. 2014;67:897-903.
10. Annual Report 2017-18. Ministry of Health and Family Welfare, Government of India. Available at: https://mohfw.gov.in. Accessed on 10 Jan 2020.

11. Kansal A, Kandpal SD. India's Story of Reducing Maternal Mortality-Achievement so far and Commitments Ahead. Indian J Comm Health. 2018;30(2):107-10.

12. Kumutha J, Chitra N, Vidyasagar D. Impact of implementation of NRHM program on NMR in Tamil Nadu (TN): a case study. Indian J Pediatr. 2014;81(12):1358-66.

13. Shah P, Shah S, Kutty RV, Modi D. Changing epidemiology of maternal mortality in rural India: time to reset strategies for MDG-5. Trop Med Int Health. 2014;19(5):568-75.

14. Gupta SK, Pal DK, Tiwari R, Garg R, Shrivastava AK, Sarawagi R et al. Impact of Janani Suraksha Yojana on institutional delivery rate and maternal morbidity and mortality: an observational study in India. J Health Popul Nutr. 2012;30(4):464-71.

15. Randive B, Diwan V, De Costa A. India's Conditional Cash Transfer Programme (the JSY) to Promote Institutional Birth: Is There an Association between Institutional Birth Proportion and Maternal Mortality? PLoS one. 2013;8(6):e67452.

16. Randive B, San Sebastian M, De Costa A, Lindholm L. Inequalities in institutional delivery uptake and maternal mortality reduction in the context of cash incentive program, Janani Suraksha Yojana: results from nine states in India. Soc Sci Med. 2014;123:1-6.

17. Mahala U, Mehta S. Maternal health outcomes following the implementation of Janani Shishu Suraksha Yojana (JSSY) at a tertiary healthcare center in Rajasthan. Int J Gynaecol Obstet. 2017;139(3):297300 .

18. Kumar V, Kumar A, Das V, Srivastava NM, Baqui $\mathrm{AH}$, Santosham $\mathrm{M}$ et al. Community-driven impact of a newborn-focused behavioral intervention on maternal health in Shivgarh, India. Int $\mathbf{J}$ Gynaecol Obstet. 2012;117(1):48-55.

19. Ghosh A. Inequality in maternal health-care services and safe delivery in eastern India. WHO South East Asia J Public Health. 2015;4(1):54-61.

20. Telecom Regulatory Authority of India, New Delhi. 2017;43. Available at: www.trai.gov.in. Accessed on 11 Jan 2020.

Cite this article as: Perumal V. Meta-analysis on maternal deaths and assessing significant factors for heterogeneity between states of India during 2016. Int J Reprod Contracept Obstet Gynecol 2021;10:3192-6. 